Original paper

\title{
Clinical audit to determine efficacy of daily infusion of low dose of $20 \%$ albumin in combination with antibiotics in patients with spontaneous bacterial peritonitis
}

\author{
Uday Sanglodkar ${ }^{1}$, Mayank Jain', Joy Vargese', Chandankumar Kedarisetty², Jayanthi Venkataraman² \\ 'Global Health City, India \\ 2SRIHER, Chennai, India
}

\begin{abstract}
Aim of the study: The recommended high dose albumin treatment for spontaneous bacterial peritonitis (SBP) is not possible in the Indian setting due to financial constraints. Aim of the study was the retrospective audit to determine the outcome of patients with SBP on combination treatment of low dose albumin and appropriate antibiotics.

Material and methods: Patients undergoing abdominal paracentesis in the period 2016-2018 were included. Patient details including age, gender, co-morbidity profile, details of previous hospitalisation and antibiotics, MELD score, and ascitic fluid analysis were noted. Details of albumin use and antibiotics were retrieved. SBP was classified based on mode of acquisition of infection and severity risk based on laboratory parameters. Statistics $\chi^{2}$ test, Mann-Whitney $U$ test, relative risk calculation. A $p$ value of $<0.05$ was considered as significant.

Results: 24 (18.8\%), 38 (29.7\%) and 66 patients (51.5\%) belonged to low, intermediate and high risk SBP groups, respectively. The median dose of albumin was $20 \mathrm{~g} /$ day for a median duration of 5 days (range 1-8). Between the 3 subgroups, there was no significant difference in the median age; the majority were men. Antibiotic escalation was necessary in intermediate and high risk cases ( $42.1 \%$ vs. $84.8 \%, p<0.0001)$. The mortality rate in intermediate and high risk groups was $29 \%$ and $42 \%$, respectively $(p=0.18)$. Between the 2 subgroups of intermediate risk, patients with serum bilirubin $<4 \mathrm{mg} / \mathrm{dl}$ and serum creatinine $>1 \mathrm{mg} / \mathrm{dl}$ were significantly older (54 vs. 49 years, $p=0.02$ ), and had high mortality $(40.7 \%$ vs. $0 \%$ ). Cirrhosis-related complications (CRC; one or more in combinations) were more frequent in high risk and intermediate risk patients $(p=0.001)$ with a 7-8 times higher risk of mortality compared to those who had no CRC.

Conclusions: Our protocol is associated with high mortality in intermediate and high risk SBP patients. Presence of one or more CRC increases the risk of mortality several fold.
\end{abstract}

Key words: ascites, liver cirrhosis, spontaneous bacterial peritonitis.

Address for correspondence

Dr. Mayank Jain, Senior Consultant, 297, Indrapuri, Indore, Madhya Pradesh, India-452017, e-mail: mayank4670@rediffmail.com

\section{Introduction}

Spontaneous bacterial peritonitis (SBP) is defined as bacterial infection of ascitic fluid in absence of an intraabdominal source of infection or malignancy [1-4]. It denotes poor prognosis in decompensated liver disease and is associated with a mortality of $30 \%$ during the first episode, and 66\% (range 30-90\%) at the end of one year. Clinicians the world over consider ascitic fluid polymorphonuclear count $(\mathrm{PMN})>250 / \mathrm{mm}^{3}$ as adequate to diagnose SBP [5].

The primary treatment of these patients is administration of intravenous antibiotics. Studies have shown that liver dysfunction (bilirubin $>4 \mathrm{mg} / \mathrm{dl}$ ) and renal 
function abnormality (blood urea $>30 \mathrm{mg} / \mathrm{dl}$ ) are predictors of mortality despite adequate antibiotic treatment [6]. In such high risk patients, administration of intravenous human albumin $1.5 \mathrm{~g} / \mathrm{kg}$ on day 1 and $1 \mathrm{~g} / \mathrm{kg}$ on day 3 has been found to be beneficial. It reduces the frequency of renal dysfunction and decreases three-month mortality from $29 \%$ to $10 \%$ [6]. Chen et al. reported that administration of a dose of $50 \mathrm{ml}$ of $20 \%$ intravenous albumin along with antibiotics once a day for 3 days reduced pro-inflammatory cytokines compared to use of antibiotics alone [7].

In an Indian setting, the dose of albumin for management of SBP is not known. It has been the practice at our centre since 2009 to use $100 \mathrm{ml}$ of $20 \%$ albumin for 3 to a maximum of 5 days i.e. $20 \mathrm{~g}$ per day. In presence of renal dysfunction or sepsis, the albumin infusion was extended to 7 days. Albumin was combined with antibiotics from day 1 . The choice of antibiotic has been piperacillin tazobactam $4.5 \mathrm{~g}$ three times a day with subsequent revision based on the culture and sensitivity report. Further switch over to meropenem or addition of tigecycline was considered when there was no response to the specific antibiotic and culture was negative. The use of piperacillin tazobactam as an empirical antibiotic for SBP, instead of third generation cephalosporins, is based on our earlier data [8]. Ours is a tertiary referral centre for liver transplant and the majority of the patients coming to our centre have been treated at outside hospitals. Thus, the prevalence of nosocomial and health care associated infection is higher than community acquired infections.

We performed a retrospective audit to determine the outcome of patients admitted with a diagnosis of SBP with or without other cirrhosis-related complications (CRC) between $1^{\text {st }}$ September 2016 and $31^{\text {st }} \mathrm{Au}$ gust 2018 using this protocol at our centre.

\section{Material and methods}

Patients undergoing abdominal paracentesis during the study period were included in the study. Of a total of 620 paracenteses, 128 patients had SBP (20.6\%).

Inclusion criteria: age more than 18 years and belonging to either gender, confirmed cirrhosis with ascites, any aetiology, with or without cirrhosis-related complications such as sepsis, renal dysfunction, hepatic encephalopathy and variceal bleeding.

Exclusion criteria: secondary cause for ascites, cirrhotic ascites with partial small bowel obstruction or perforated viscus.

Patient details included age, gender and co-morbidity profile. Details of previous hospitalisation and antibiotic received were obtained from inpatient medical re- cords. Baseline laboratory tests included blood counts, serum electrolytes, serum creatinine and liver biochemistry. Model for end-stage liver disease (MELD) and Child-Turcotte-Pugh (CTP) scores at each admission and details of blood culture, ascitic fluid cell count, culture, albumin and protein were noted. Choice of antibiotic, dosage and duration, details of albumin infusion, its concentration, number of days administered were also retrieved from the hospital records.

Diagnosis of SBP: Ascitic fluid culture positivity (bacterascites) and/or an ascitic fluid polymorphonuclear leucocyte count greater than $250 / \mathrm{mm}^{3}(0.25$ $\times 10^{9} / 1$ ) were both considered for a diagnosis of SBP. We modified the existing criterion for diagnosis of SBP as both neutrocytic and culture positive SBP have similar clinical presentation and treatment protocol [9].

Spontaneous bacterial peritonitis was classified as:

- community acquired SBP (C-SBP): SBP occurring within 72 hours of hospitalisation [10];

- nosocomial SBP (N-SBP): SBP occurring after 72 hours of hospitalisation [11];

- health care associated SBP (H-SBP): hospitalisation in an acute care hospital or an emergency department for 2 or more days within the previous 90 days, or admitted to a nursing home [12].

We classified patients with SBP into three categories based on serum bilirubin and serum creatinine levels (Fig. 1) [13]:

- low risk: serum bilirubin $<4 \mathrm{mg} / \mathrm{dl}$, serum creatinine $<1 \mathrm{mg} / \mathrm{dl}$,

- intermediate risk (fulfilling any one criterion): serum bilirubin $>4 \mathrm{mg} / \mathrm{dl}$ and serum creatinine $<1 \mathrm{mg} / \mathrm{dl}$, serum bilirubin $<4 \mathrm{mg} / \mathrm{dl}$ and serum creatinine $>1 \mathrm{mg} / \mathrm{dl}$,

- high risk: serum bilirubin $>4 \mathrm{mg} / \mathrm{dl}$, serum creatinine $>1 \mathrm{mg} / \mathrm{dl}$.

The primary outcome of the study was worsening of the patient's symptoms or mortality during the same admission.

\section{Statistics}

The patients in three SBP risk groups were compared for age, gender, aetiology, choice of antibiotic, dose of albumin and outcome. The data were entered in a Microsoft Excel sheet. Age, duration and laboratory parameters were expressed as median and range. Other parameters were expressed as number and proportions. Comparison of medians was by the Mann-Whitney $U$ test and $\chi^{2}$ test for comparison of proportions. Impact of presence of one or more CRC on mortality was assessed using relative risk calculation. A $p$ value of $<0.05$ was considered significant. 


\section{Results}

Of the 128 patients with SBP, 24 (18.8\%), 38 (29.7\%) and 66 patients $(51.5 \%)$ belonged to low, intermediate and high risk groups, respectively (Table 1). Based on the mode of acquisition of infection, it was noted that H-SBP was the commonest $(61,47.7 \%)$ followed by C-SBP $(52,40.6 \%)$ and N-SBP $(15,11.7 \%)$. Ascitic culture positive SBP infection was noted in 7 cases and the rest had culture negative neutrocytic ascites. Three of these cases had N-SBP and the remaining 4 had H-SBP. Escherichia coli (3), Klebsiella (1), Acinetobacter (1), Salmonella paratyphi (1) and Staphylococcus aureus (1) were the ascitic fluid isolates.

Associated cirrhosis-related complications in our 128 patient cohort included - variceal bleeding ( 2 cases), hepatic encephalopathy $(44,34.4 \%)$, renal dysfunction (oliguria/rise in creatinine) $(48,37.5 \%)$ and nonSBP infections such as cellulitis, pneumonia, urinary tract infections, etc. $(9,28.2 \%)$, which were noted in 58 patients.

The median dose of albumin administered was $100 \mathrm{ml}$ (20\%), i.e. $20 \mathrm{~g} /$ day for a median duration of 5 days (range 1-8). All patients received piperacillin tazobactam as the initial choice antibiotic. Following the ascetic \pm blood culture reports and clinical response at 72 hours, 72 patients $(56.3 \%)$ in intermediate or high risk groups required a switch over to tigecycline or meropenem. Thirty-nine $(30.5 \%)$ patients expired during the same admission.

\section{Comparison between high, intermediate and high risk groups}

Between the 3 subgroups, there was no significant difference in the median age; the majority were men.

In the low risk group, community acquired infection was the most common mode of acquisition of infection; median MELD was significantly lower than in the other two groups. None of the patients in this group required escalation of antibiotic therapy; none had an adverse effect to albumin. There were no deaths.

$\mathrm{H}$-SBP was more frequent in intermediate risk and high risk patients. N-SBP $(15,22.8 \%)$ was significant in high risk cases.

Though the median dose and duration of albumin were similar in all three groups, antibiotic escalation was necessary in intermediate and high risk cases (42.1\% vs. $84.8 \%, p<0.0001)$. The mortality rate in intermediate and high risk groups was $29 \%$ and $42 \%$, respectively $(p=0.18)$.

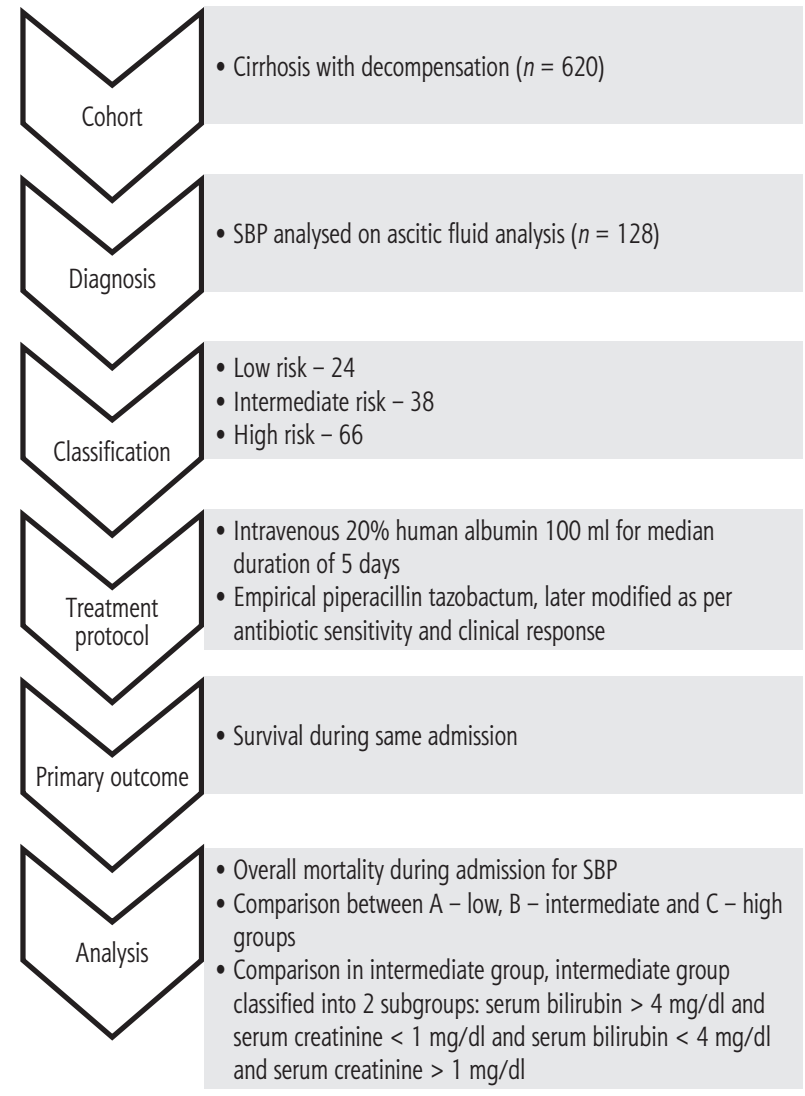

Fig. 1. Protocol for management of patients with SBP

\section{Comparison of two subgroups of intermediate risk}

Between the 2 subgroups of intermediate risk (Table 2), patients with serum bilirubin $<4 \mathrm{mg} / \mathrm{dl}$ and high serum creatinine i.e. $>1 \mathrm{mg} / \mathrm{dl}$ were significantly older (54 vs. 49 years, $p=0.02$ ), and with a high mortality ( $40.7 \%$ vs. $0 \%$ ). Though $51.4 \%$ of these cases required antibiotic escalation compared to $18.4 \%$ in those with high serum bilirubin and normal creatinine, the difference was not statistically significant $(p=0.06)$.

Overall, there were no major adverse effects of human albumin infusion except for chest congestion in a small proportion of patients.

\section{Impact of CRC on outcome}

Various CRC such as variceal bleeding, hepatic encephalopathy, other non-SBP infections and renal dysfunction were evaluated for impact on outcome of patients. We noted that these CRC (one or more in combinations) were more frequent in high risk and intermediate risk patients $(p=0.001)$. However, in the two subgroups of intermediate risk patients, there was no statistically significant difference in the occurrence of CRC. Presence of one or more CRC with SBP is likely to affect the outcome as noted by higher mor- 
Table 1. Comparison between high, intermediate and low risk groups

\begin{tabular}{|c|c|c|c|c|}
\hline Parameters & $\begin{array}{l}\text { Low risk group } \\
\qquad(n=24)\end{array}$ & $\begin{array}{l}\text { Intermediate group } \\
\quad(n=38)\end{array}$ & $\begin{array}{l}\text { High risk group } \\
\quad(n=66)\end{array}$ & $P$ value \\
\hline Age (median, range) & $54(31-68)$ & $52(38-72)$ & $52(33-63)$ & 0.14 \\
\hline Sex & & & & 0.0004 \\
\hline Male & $16(66.7 \%)$ & $32(84 \%)$ & $64(97 \%)$ & \\
\hline Female & $8(33.3 \%)$ & $6(16 \%)$ & $2(3 \%)$ & \\
\hline Aetiology of cirrhosis & & & & 0.20 \\
\hline Alcohol & $8(33.3 \%)$ & $19(50 \%)$ & $40(60.6 \%)$ & \\
\hline Hepatitis B & $4(16.7 \%)$ & $2(5.25 \%)$ & $6(9.1 \%)$ & \\
\hline Hepatitis C & 0 & $2(5.25 \%)$ & $2(3 \%)$ & \\
\hline Cryptogenic & $12(50 \%)$ & $15(39.5 \%)$ & $18(27.3 \%)$ & \\
\hline Diabetes mellitus & $6(25 \%)$ & $13(34.2 \%)$ & $24(36.7 \%)$ & 0.59 \\
\hline Type of SBP & & & & $<0.0001$ \\
\hline Community acquired & $22(91.6 \%)$ & $18(47.4 \%)$ & $12(18.2 \%)$ & \\
\hline Health care associated & $2(8.4 \%)$ & $20(52.6 \%)$ & $39(59 \%)$ & \\
\hline Nosocomial & 0 & 0 & $15(22.8 \%)$ & \\
\hline Cirrhosis-related complications & & & & 0.001 \\
\hline None & $21(87.5 \%)$ & $20(52.6 \%)$ & $29(44 \%)$ & \\
\hline One or more & $3(12.5 \%)$ & $18(47.4 \%)$ & $37(56 \%)$ & \\
\hline Baseline serum creatinine (mg/dl) & & & & 0.03 \\
\hline Median, range & $0.7(0.5-0.9)$ & $1.7(0.7-4.6)$ & $1.9(1.2-5.3)$ & \\
\hline Baseline serum bilirubin (mg/dl) & & & & 0.001 \\
\hline Median, range & $2.3(1.9-3.9)$ & $4.6(1.8-22.6)$ & $9.7(4.3-32.8)$ & \\
\hline Baseline MELD & & & & 0.04 \\
\hline Median, range & $19(13-24)$ & $23(16-36)$ & $24(15-40)$ & \\
\hline Baseline serum sodium (meq/l) & & & & 0.23 \\
\hline Median, range & $133(126-140)$ & $131(114-147)$ & $132(118-150)$ & \\
\hline Duration of albumin given (days) & & & & 0.56 \\
\hline Median, range & $5(3-6)$ & $5(1-8)$ & $5(3-7)$ & \\
\hline Adverse effects of albumin & None & Chest congestion in 4 & Chest congestion in 5 & NA \\
\hline Antibiotic escalation & None & $16(42.1 \%)$ & $56(84.8 \%)$ & NA \\
\hline Outcome & & & & NA \\
\hline Recovered & $24(100 \%)$ & $27(71 \%)$ & $38(58 \%)$ & \\
\hline Expired & $0(0 \%)$ & $11(29 \%)$ & $28(42 \%)$ & \\
\hline
\end{tabular}

tality in high and intermediate risk patients. Relative risk of death in those with at least one CRC in high risk and intermediate risk groups was 8.82 (3.47-22.41, $p<0.0001)$ and $7.36(2.44-22.18, p=0.0004)$ respectively compared to those who had no CRC.

\section{Discussion}

The present study showed that the majority of our patients with decompensated liver disease complicat- ed by SBP belonged to the high risk group. Mortality during the same admission was nil in the low risk group and $29 \%$ and $42 \%$ respectively in intermediate and high risk groups of patients. The recommended dose of albumin in SBP in our patients is based on an earlier study by Sort et al. [6] where the authors noted that patients given antibiotics along with albumin had significantly lower renal impairment and mortality compared to those given antibiotics alone. A more recent study by Xue et al. [14] reported that antibiotics 
Table 2. Comparison of 2 groups of patients in intermediate group

\begin{tabular}{|c|c|c|c|}
\hline Parameters & $\begin{array}{c}\text { Group } A(n=11) \\
\text { Serum bilirubin }>4 \mathrm{mg} / \mathrm{dl} \\
\text { Serum creatinine }<1 \mathrm{mg} / \mathrm{dl}\end{array}$ & $\begin{array}{c}\text { Group } B(n=27) \\
\text { Serum bilirubin }<4 \mathrm{mg} / \mathrm{dl} \\
\text { Serum creatinine }>1 \mathrm{mg} / \mathrm{dl}\end{array}$ & $P$ value \\
\hline Age (median, range) & $49(38-55)$ & $54(38-72)$ & 0.02 \\
\hline Sex & & & 0.46 \\
\hline Male & $10(90.9 \%)$ & $22(81.5 \%)$ & \\
\hline Female & $1(9.1 \%)$ & $5(18.5 \%)$ & \\
\hline Aetiology of cirrhosis & & & 0.04 \\
\hline Alcohol & $8(72.7 \%)$ & $11(40.7 \%)$ & \\
\hline Hepatitis B/hepatitis C & $2(18.2 \%)$ & $2(7.4 \%)$ & \\
\hline Cryptogenic & $1(9.1 \%)$ & $14(51.8 \%)$ & \\
\hline Diabetes mellitus & $2(18.2 \%)$ & $11(40.7 \%)$ & 0.20 \\
\hline Type of SBP & & & 0.19 \\
\hline Community acquired & $7(63.6 \%)$ & $11(40.7 \%)$ & \\
\hline Health care associated & $4(36.4 \%)$ & $16(59.3 \%)$ & \\
\hline Cirrhosis-related complications & & & 0.57 \\
\hline None & $5(45.5 \%)$ & $15(55.5 \%)$ & \\
\hline One or more & $6(54.5 \%)$ & $12(44.5 \%)$ & \\
\hline Baseline MELD & & & 0.43 \\
\hline Median, range & $23(19-28)$ & $23(16-36)$ & \\
\hline Serum sodium (meq/l) & & & 0.23 \\
\hline Median, range & $130(114-136)$ & $132(118-147)$ & \\
\hline Duration of albumin given in days & & & 0.67 \\
\hline Median, range & $5(3-7)$ & $5(1-8)$ & \\
\hline Antibiotic escalation & $2(18.4 \%)$ & $14(51.8 \%)$ & 0.06 \\
\hline Adverse effects of albumin & & & 0.33 \\
\hline Chest congestion & $2(18.2 \%)$ & $2(7.4 \%)$ & \\
\hline Outcome & & & NA \\
\hline Recovered & $11(100 \%)$ & 16 (59.3\%) & \\
\hline Expired & 0 & $11(40.7 \%)$ & \\
\hline
\end{tabular}

along with human albumin in a lower dose $(0.5 \mathrm{~g} / \mathrm{kg}$ to $1.0 \mathrm{~g} / \mathrm{kg}$ within $6 \mathrm{~h}$ of enrolment, the same amount on day 3 , once every 3 days for a total of 3 weeks) also reduced the incidence of renal failure and mortality. In our cohort, we noted that the overall mortality was $30.5 \%$ and in the high risk group of SBP patients it was $42 \%$. The cumulative dose of albumin administered was $80 \mathrm{~g}$ (range, 60-540 g). The higher mortality in our cohort compared to previous studies may be related to more patients being in high risk category frequent nosocomial or health care associated infections (104, $81.25 \%$ ) or a lower dose of albumin. A recent systematic analysis by Jamtgaard et al. reported that albumin administration within 6 hours of diagnosis resulted in reduced renal dysfunction and mortality [15].
Studies from other centres from India have shown variable prevalence of C-SBP $[16,17]$. The reported mortality at one month and six month ranges from $23.5 \%$ to $59 \%$ respectively. Our centre is a tertiary care centre where the majority of the patients are referred for liver transplantation. Thus, the incidence of nosocomial and health care associated infections is high. A previous study from our centre on culture isolates and antibiotic sensitivity pattern in cirrhotic patients with infections showed that the majority of infections in our centre were due to Klebsiella followed by Escherichia coli and Enterococcus in nosocomial and health care associated infections. However, Enterococcus was followed by E. coli and Klebsiella in community acquired infections. The most common resistant strains 
were extended-spectrum $\beta$-lactamase (ESBL)-producing Enterobacteriaceae followed by carbapenemaseproducing Klebsiella and methicillin-resistant Staphylococcus aureus [8]. Thus, we prefer to use piperacillin tazobactam as empirical antibiotic therapy rather than third generation cephalosporins.

The present study highlights the use of low dose albumin therapy. We have used a lower daily dose of albumin for a median duration of 5 days for SBP patients. Though this dose is not recommended in western literature, it is frequently used in our setting, often due to monetary constraints. We do not have similar information regarding the use of this low dose albumin protocol from other tertiary referral centres across the Indian subcontinent. Side effects to even this low dose albumin therapy were noted in 9 cases $(8.7 \%)$ in intermediate and high risk groups. In patients with cirrhosis and SBP, renal function frequently becomes impaired due to a reduction in effective arterial blood volume. The effectiveness of our low dose albumin protocol compared to the recommended dosage protocol needs to be assessed prospectively in randomised controlled trials.

We noted that in the intermediate group, patients with higher creatinine $>1 \mathrm{mg} / \mathrm{dl}$ but low bilirubin $<4 \mathrm{mg} / \mathrm{dl}$ had a worse outcome. This suggests the importance of renal dysfunction in predicting worse outcome in patients with end stage liver disease and with SBP. A recent review highlighted independent predictors of mortality in patients with SBP. These include severity of liver dysfunction as indicated by serum bilirubin $>4 \mathrm{mg} / \mathrm{dl}$, renal dysfunction, nosocomial source of SBP, severe sepsis as indicated by culture positivity of both blood and ascitic fluid and hemodynamic instability [18]. The relative risk of mortality in our patients with at least one CRC in high and intermediate risk groups of SBP was nearly 7-8 times higher compared to those who had no CRC.

\section{Limitations}

The present study is a retrospective analysis and has few patients in the C-SBP group. It was not possible to obtain information on the time lag between the diagnosis of SBP and institution of human albumin. Several patients in our cohort had one or more CRCs. The impact of these on SBP-related mortality cannot be ruled out. The effect of acute kidney injury could not be assessed as a one-time value of serum creatinine was taken for classification of patients and serial measurements were not taken. Moreover, differentiation between decompensation of cirrhosis and acute-onchronic liver failure was not done. We did not evaluate the prevalence of acute kidney injury and acute-onchronic liver cell failure in the study cohort.

In conclusion, administration of $20 \mathrm{~g}$ albumin per day along with antibiotics is a valid alternative instead of the recommended high dose albumin of $1.5 \mathrm{~g} / \mathrm{kg}$ on day 1 and $1 \mathrm{~g} / \mathrm{kg}$ on day 3 . Patients with renal impairment had a worse outcome than those without, irrespective of the dose of albumin given. Further multicentric studies need to be done to define the dose of albumin to be given in SBP which is cost-effective and, more importantly, improves haemodynamics and reduces renal impairment.

\section{Disclosure}

The authors report no conflict of interest.

\section{References}

1. Caly WR, Strauss E. A prospective study of bacterial infections in patients with cirrhosis. J Hepatol 1993; 18: 353-358.

2. Evans LT, Kim WR, Poterucha JJ, Kamath PS. Spontaneous bacterial peritonitis in asymptomatic outpatients with cirrhotic ascites. Hepatology 2003; 37: 897-901.

3. Nobre SR, Cabral JE, Gomes JJ, Leitão MC. In-hospital mortality in spontaneous bacterial peritonitis: a new predictive model. Eur J Gastroenterol Hepatol 2008; 20: 1176-1181.

4. Wiest R, Krag A, Gerbes A. Spontaneous bacterial peritonitis: recent guidelines and beyond. Gut 2012; 61: 297-310.

5. Runyon BA; AASLD Practice Guidelines Committee. Management of adult patients with ascites due to cirrhosis: an update. Hepatology 2009; 49: 2087-2107.

6. Sort P, Navasa M, Arroyo V, et al. Effect of intravenous albumin on renal impairment and mortality in patients with cirrhosis and spontaneous bacterial peritonitis. N Engl J Med 1999; 341: 403-409.

7. Chen TA, Tsao YC, Chen A, et al. Effect of intravenous albumin on endotoxin removal, cytokines, and nitric oxide production in patients with cirrhosis and spontaneous bacterial peritonitis. Scand J Gastroenterol 2009; 44: 619-625.

8. Jain M, Varghese J, Michael T, et al. An insight into antibiotic resistance to bacterial infection in chronic liver disease. J Clin Exp Hepatol 2017; 7: 305-309.

9. Runyon BA, Hoefs JC. Culture-negative neutrocytic ascites: a variant of spontaneous bacterial peritonitis. Hepatology 1984; 4: 1209-1211.

10. Chon YE, Kim SU, Lee CK, et al. Community-acquired vs. nosocomial spontaneous bacterial peritonitis in patients with liver cirrhosis. Hepatogastroenterology 2014; 61: 2283-2290.

11. Venditti M, Falcone M, Corrao S, et al. Study Group of the Italian Society of Internal Medicine (outcomes of patients hospitalized with community-acquired, healthcare associated and hospital-acquired pneumonia). Ann Intern Med 2009; 150: 19-26.

12. Merli M, Lucidi C, Giannelli V, et al. Cirrhotic patients are at risk for health care-associated bacterial infections. Clin Gastroenterol Hepatol 2010; 8: 979-985.

13. Sigal SH, Stanca CM, Fernandez J, et al. Restricted use of albumin for spontaneous bacterial peritonitis. Gut 2007; 56: 597-599.

14. Xue HP, LinB, Zhong J, et al. Effect of albumin infusion on preventing the deterioration of renal function in patients with 
spontaneous bacterial peritonitis. Chinese J Dig Dis 2002; 32-34.

15. Jamtgaard L, Manning L, Cohn B, et al. Does albumin infusion reduce renal impairment and mortality in patients with SBP. Ann Emerg Med 2016; 67: 458-459.

16. Balaraju G, Patil M, Krishnamurthy AC, et al. Comparative study of community acquired and nosocomial spontaneous bacterial peritonitis and its variants in 150 patients. J Clin Exp Hepatol 2017; 7: 215-221.

17. Baijal R, Amarapurkar D, Praveen Kumar HR, et al. A multicenter prospective study of infections related morbidity and mortality in cirrhosis of liver. Indian J Gastroenterol 2014; 33: 336-342.

18. Shalimar, Acharya SK. Difficult to treat spontaneous bacterial peritonitis. Trop Gastroenterol 2013; 34: 7-13. 